\title{
Hypocretin/orexin signaling in the hypothalamic paraventricular nucleus is essential for the expression of nicotine withdrawal
}

\author{
Ainhoa Plaza-Zabala, África Flores, Rafael Maldonado and Fernando Berrendero ${ }^{\#}$ \\ Laboratory of Neuropharmacology, Department of Experimental and Health Sciences, \\ University Pompeu Fabra, PRBB, C/ Doctor Aiguader 88, 08003 Barcelona, Spain \\ ${ }^{\#}$ Corresponding author: Fernando Berrendero, Phone: +34-93-3160890; Fax: + 34-93- \\ 3160901; E-mail: fernando.berrendero@upf.edu
}

Keywords: nicotine; hypocretin; withdrawal; SB334867; TCSOX229; PVN

Number of words (abstract): 251

Number of words (text): 3872

Number of figures: 4

Number of tables: 1

Supplementary material: 1 (Methods, and 4 Figures) 


\begin{abstract}
Background: Hypocretin (orexin) signaling is involved in drug addiction. In this study, we investigated the role of these hypothalamic neuropeptides in nicotine withdrawal by using behavioral and neuroanatomical approaches.
\end{abstract}

Methods: Nicotine withdrawal syndrome was precipitated by mecamylamine $(2 \mathrm{mg} / \mathrm{kg}$, sc) in C57BL/6J nicotine dependent mice (25 mg/kg/day for 14 days) pretreated with the hypocretin receptor 1 (Hcrtr-1) antagonist SB334867 (5 and $10 \mathrm{mg} / \mathrm{kg}$, ip), the hypocretin receptor 2 (Hcrtr-2) antagonist TCSOX229 (5 and $10 \mathrm{mg} / \mathrm{kg}$, ip), and in prepro-hypocretin knockout $(\mathrm{KO})$ mice. c-Fos expression was analyzed in several brain areas related to nicotine dependence by immunofluorescence techniques. Retrograde tracing with rhodamine-labelled fluorescent latex microspheres was used to determine whether the hypocretin neurons project directly to the paraventricular nucleus of the hypothalamus (PVN), and SB334867 was locally administered intra-PVN (10 $\mathrm{nmol} / \mathrm{side}$ ) to test the specific involvement of Hcrtr-1 in this brain area during nicotine withdrawal.

Results: Somatic signs of nicotine withdrawal were attenuated in mice pretreated with SB334867, and in prepro-hypocretin KO mice. No changes were found in TCSOX229 pretreated animals. Nicotine withdrawal increased the percentage of hypocretin cells expressing c-Fos in the perifornical, dorsomedial and lateral hypothalamus. In addition, the increased c-Fos expression in the PVN during withdrawal was dependent on hypocretin transmission through Hcrtr-1 activation. Hypocretin neurons directly innervate the PVN and the local infusion of SB334867 into the PVN decreased the expression of nicotine withdrawal. 
Conclusions: These data demonstrate that hypocretin signaling acting on Hcrtr-1 in the PVN plays a crucial role in the expression of nicotine withdrawal. 


\section{Introduction}

Tobacco consumption is one of the main public health problems worldwide and represents the leading cause of preventable deaths in most developed countries. Despite the harmful effects of tobacco smoking, relapse rates are extremely high even with the help of the available medications and support systems (1). When a person quits smoking, nicotine withdrawal symptoms appear and this syndrome might have an important role in the relapse of tobacco consumption (2). Thus, cessation of tobacco intake in humans precipitates both somatic and motivational symptoms of withdrawal, which may include severe craving for nicotine, irritability, anxiety, loss of concentration, bradycardia, gastrointestinal discomfort, depressed mood, impatience, insomnia, increased appetite and weight gain $(3,4)$. In rodents, nicotine withdrawal is also characterized by the manifestation of both somatic signs and affective changes similar to those reported in humans. The somatic signs include teeth chattering, palpebral ptosis, tremor, wet dog shakes, changes in locomotor activity and other behavioral effects $(5,6)$, while the affective changes consist of increased anxiety-like behavior, aversive effects and reward deficits $(7,8)$. The mechanisms and brain regions underlying the somatic manifestation of nicotine abstinence are still poorly understood (9), although an important role for the habenulo-interpeduncular system in the mediation of the somatic signs of withdrawal has been recently reported (10).

Hypocretin-1 and -2 (also known as orexin A and B) are lateral hypothalamic neuropeptides that project throughout the brain $(11,12)$, and produce their responses by the activation of two G-protein coupled receptors called hypocretin receptor-1 and -2 (Hcrtr-1 and Hcrtr-2). Although hypocretins play an important role in narcolepsy and sleep $(13,14)$, more recent work has highlighted the relevance of these neuropeptides in the regulation of reward and drug dependence (15). Specifically, emerging evidence 
indicates that hypocretin transmission is involved in the addictive properties of nicotine (16,17). Thus, the Hcrtr-1 antagonist SB334867 (18) and the mixed Hcrtr-1/Hcrtr-2 antagonist almorexant (19) decreased nicotine self-administration behavior in rats. A similar reduction of nicotine self-administration was observed when infusing SB334867 directly into the insular cortex (18). We have also recently shown a role for hypocretins in the processes driving to relapse to nicotine-seeking. Accordingly, intracerebroventricular (icv) infusion of hypocretin-1 reinstated a previously extinguished nicotine-seeking behavior in mice (20), through a mechanism independent of corticotrophin releasing factor (CRF) activation.

Here, we investigate the specific contribution of hypocretins in another critical component of nicotine addiction, the manifestations of the nicotine withdrawal syndrome. For this purpose, both behavioral and biochemical techniques were used to establish the role of these hypothalamic neuropeptides in the somatic expression of nicotine withdrawal. 


\section{Methods and Materials}

\section{Animals}

Experiments were performed in male C57BL/6J mice (Charles River, L'Arbresle, France) and in male prepro-hypocretin knockout (KO) and their wild-type C57BL/6J mice (8-10 week-old). Generation of mice with a deletion of the prepro-hypocretin gene has been previously described (21). These mice do not produce either of the hypocretin1 and -2 peptides and have been extensively characterized at the behavioral level. Thus, KO mice display fragmented sleep and behavioral arrests reminiscent of cataplexy during the dark period, but otherwise normal sleep homeostasis (22). Under ad libitum conditions, feeding and drinking patterns are also normal. Prepro-hypocretin KO mice were backcrossed for 9 generations into C57BL/6J, and genotyped as described elsewhere (21). Mice were housed five per cage in a temperature- $\left(21 \pm 1^{\circ} \mathrm{C}\right)$ and humidity- (55 $\pm 10 \%)$ controlled room with a 12-h/12-h light/dark cycle (light between 08:00 to 20:00). Mice were single housed after surgery. All surgical and experimental procedures were performed during the light phase. Food and water were available ad libitum except during the exposure to the different behavioral paradigms. Mice were habituated to their new environment and handled for 1 week after arrival, and before starting the experimental procedure. The observer was blind to treatment in all the experiments. Animal procedures were conducted in accordance with the guidelines of the European Communities Directive 86/609/EEC regulating animal research and approved by the local ethical committee (CEEA-IMAS-UPF). 


\section{Drugs}

(-)-Nicotine hydrogen tartrate salt [(-)-1-methyl-2(3-pyridyl)pyrrolidine] (Sigma) was dissolved in physiological saline $(0.9 \% \mathrm{NaCl})$ and administered at the dose of 25 $\mathrm{mg} / \mathrm{kg} /$ day by using subcutaneously implanted osmotic minipumps. Nicotine dose was calculated as nicotine hydrogen tartrate salt. Mecamylamine hydrochloride $(2 \mathrm{mg} / \mathrm{kg})$ (Sigma) was dissolved in physiological saline $(0.9 \% \mathrm{NaCl})$ and administered by subcutaneous route (sc) in a volume of $10 \mathrm{ml} / \mathrm{kg}$ body weight. The Hcrtr-1 antagonist SB334867 (5 and $10 \mathrm{mg} / \mathrm{kg}$ ) (Tocris) was dissolved in 1\% (w/v) 2-Hydroxypropyl- $\beta$ cyclodextrin (Sigma) and 10\% DMSO (v/v) in distilled water, and administered by intraperitoneal route (ip) in a volume of $5 \mathrm{ml} / \mathrm{kg}$ body weight. The SB334867 doses used were based on previous studies and did not induce changes in locomotor activity (20). The Hcrtr-2 antagonist TCSOX229 (5 and $10 \mathrm{mg} / \mathrm{kg}$ ) (Tocris) was dissolved in physiological saline $(0.9 \% \mathrm{NaCl})$, and administered by ip route in a volume of $5 \mathrm{ml} / \mathrm{kg}$ body weight. The TCSOX229 doses were based on a pilot experiment testing the effects of this antagonist on the hyperactivity induced by hypocretin- 2 in C57BL/6J mice (Figure S1 in Supplement 1). Hypocretin-2 and colchicine (Sigma) were dissolved in physiological saline and administered by icv route. For intra-hypothalamic paraventricular nucleus (intra-PVN) administration, SB334867 (10 nmol/side) was dissolved in a 50:50 sterile saline solution:DMSO vehicle. This dose was based on preceding reports $(18,23)$. Ketamine hydrochloride $(100$ mg/kg) (Imalgène 1000; Rhône Mérieux, Lyon, France) and xylazine hydrocloride (20 mg/kg) (Sigma) were mixed and dissolved in ethanol (5\%) and distilled water (95\%). This anaesthetic mixture was administered ip in a volume of $10 \mathrm{ml} / \mathrm{kg}$ body weight. 


\section{Nicotine treatment and withdrawal}

Nicotine dependence was induced by using Alzet osmotic minipumps (Model 2002) (Alzet $^{\circledR}$, Cupertino, CA, USA). These minipumps, implanted subcutaneously under brief isofluorane anaesthesia, contained saline or nicotine solutions and delivered a constant subcutaneous flow in a rate of $0.5 \mu \mathrm{l} / \mathrm{h}$ during the whole experimental sequence. The concentration of nicotine was adjusted to compensate for differences in the body weight of the mice. Thus, the average-weighed mice received a dose of approximately $25 \mathrm{mg} / \mathrm{kg} /$ day of nicotine during 14 days. Animals were daily handled during the complete experimental sequence to avoid c-Fos activation under basal conditions. Nicotine withdrawal syndrome was precipitated 14 days after minipump implantation by injection of the nicotinic receptor antagonist, mecamylamine $(2 \mathrm{mg} / \mathrm{kg}$, sc). Pharmacological (SB334867 and TCSOX229 administration) and genetic (preprohypocretin KO mice) approaches were used in this set of experiments. For the pharmacological studies, mice received a systemic (5 and $10 \mathrm{mg} / \mathrm{kg}$, ip) injection of SB334867 or TCSOX229, or intra-PVN (10 nmol/side) infusion of SB334867 $30 \mathrm{~min}$ before mecamylamine-precipitated nicotine withdrawal syndrome. The somatic signs of withdrawal were evaluated immediately after mecamylamine injection during a period of 25 min. The number of wet dog shakes, front paw tremors and scratches was counted. Body tremor, ptosis, teeth chattering, genital licks and piloerection were scored 1 for appearance or 0 for non-appearance within each $5 \mathrm{~min}$ time. The locomotor activity over 5 min periods was rated 0,1 or 2 ( 0 for inactivity, 1 for low activity and 2 for normal activity). A global withdrawal score was calculated for each animal by giving each individual sign a relative weight as previously reported (6): 0.5 for each episode of wet dog shake, front paw tremor, and scratching; 1 for the presence of ptosis, genital licks, body tremor, piloerection and teeth chattering, each observation period of 
$5 \mathrm{~min}$. The relative weight of locomotor activity for each 5 min period was 0 for normal activity, 0.5 for low activity and 1 for inactivity.

\section{Cannula implantation and intra-PVN infusion of SB334867}

See Supplement 1

\section{Locomotor activity}

See Supplement 1

\section{Retrograde axonal tracing}

See Supplement 1

\section{Immunofluorescence studies}

See Supplement 1

\section{Data analysis}

Two-way ANOVA was used to analyze the global withdrawal score and c-Fos expression alone or in combination with hypocretin-1, CRF and arginine-vasopressin (AVP) using SB334867 or TCSOX229 pretreatment/genotype and treatment as between subject factors of variation. Subsequent post-hoc analysis (Newman-Keuls or Fisher LSD test) was used when required. The percentage of hypocretin neurons projecting to the PVN from the perifornical and dorsomedial hypothalamus (PFA/DMH) and lateral hypothalamus (LH) was compared by a paired Student's $t$ test. The level of significance was $p<0.05$ in all experiments. 


\section{Results}

\section{Hypocretin transmission through the activation of hypocretin receptor-1 modulates the expression of nicotine withdrawal}

First, we investigated the role of hypocretin transmission in regulating the expression of the nicotine withdrawal syndrome. During the behavioral observation performed before mecamylamine administration, no somatic signs of withdrawal were observed in any group of animals. After mecamylamine injection, nicotine withdrawal syndrome was manifested by the presence of a variety of somatic signs in mice receiving chronic nicotine perfusion. Prepro-hypocretin KO mice showed a reduction in the severity of the withdrawal syndrome. Thus, a significant interaction between nicotine treatment and genotype $\left(F_{1,45}=8.77, p<0.01\right)$ was revealed for the global withdrawal score by twoway ANOVA. Post-hoc analysis confirmed that the nicotine withdrawal syndrome was suppressed in prepro-hypocretin KO mice $(p<0.05)$ (Figure 1A). Similar results were obtained in animals pretreated with the Hcrtr-1 antagonist SB334867 (5 and $10 \mathrm{mg} / \mathrm{kg}$, ip) before mecamylamine. Thus, two-way ANOVA calculated for global withdrawal score revealed a significant interaction between SB334867 and nicotine treatment $\left(F_{2,85}\right.$ $=3.91, p<0.05)$. Subsequent post-hoc analysis showed a reduction of the somatic nicotine withdrawal in SB334867 pretreated mice $(5 \mathrm{mg} / \mathrm{kg}: p<0.05 ; 10 \mathrm{mg} / \mathrm{kg}: p<$ 0.01) (Figure 1B). In contrast, pretreatment with the Hcrtr-2 antagonist TCSOX229 (5 and $10 \mathrm{mg} / \mathrm{kg}$, ip) did not modify the severity of nicotine withdrawal (Figure 1C), indicating that the Hcrtr-1 plays a specific role in the modulation of this effect. Individual signs of nicotine withdrawal are shown in Table 1.

To further characterize the role of hypocretin neurons in response to nicotine withdrawal, we studied the possible activation of these cells by using double label immunofluorescence of c-Fos with hypocretin-1 in the PFA/DMH as well as in the LH. 
c-Fos is an immediaty-early gene used as a functional anatomical marker of cellular activation (24). Responses were evaluated following nicotine withdrawal with or without SB334867 (5 mg/kg, ip) or TCSOX229 (10 mg/kg, ip) pretreatment. Mecamylamine-precipitated withdrawal was accompanied by an increase in the percentage of hypocretin neurons expressing c-Fos in both the PFA/DMH and the LH (Figures $1 \mathrm{E}$ and $1 \mathrm{~F}$ ). Interestingly, in the PFA/DMH, two-way ANOVA showed a significant interaction between pretreatment and nicotine treatment $\left(F_{2,43}=3.71, p<\right.$ 0.05), and SB334867 prevented the activation of hypocretin cells induced by nicotine withdrawal in this brain structure $(p<0.05)$ suggesting a local modulation by Hcrtr-1 (Figure 1E). In the LH, two-way ANOVA revealed a significant effect of nicotine treatment $\left(F_{1,43}=13.99, p<0.001\right)$, and SB334867 did not affect this activation of hypocretin neurons (Figure 1E). TCSOX229 pretreatment did not modify the activation of hypocretin neurons neither in the LH nor the PFA/DMH (Figure 1F).

\section{Hypocretins acting on the hypocretin receptor-1 regulate the activation of the}

\section{hypothalamic paraventricular nucleus associated to nicotine withdrawal}

Hypocretin neurons have extensive projections to different brain regions that could mediate the effects of the activation observed during nicotine withdrawal. To understand better the neuroanatomic basis of the role of hypocretins in the nicotine withdrawal syndrome, we carried out immunofluorescence of c-Fos in several brain regions related to nicotine addiction. Mecamylamine-precipitated withdrawal induced an increased number of c-Fos expressing neurons in the PVN, lateral septum (LS) and the bed nucleus of the stria terminalis (BNST) (Figure 2A-2C and Figure S2 in Supplement 1). No changes were observed in other brain areas analyzed such as the basolateral amygdala, paraventricular nucleus of the thalamus, shell and core of the 
nucleus accumbens and medial septum (Figure S3 in Supplement 1). Other regions including central amygdala, striatum and hippocampus did not show detectable levels of c-Fos. Notably, c-Fos expression in the PVN, but not in the LS and the BNST, was significantly reduced in animals pretreated with SB334867 (5 mg/kg, ip) or in mice lacking the prepro-hypocretin gene. In the genetic approach, two-way ANOVA showed a significant interaction between genotype and nicotine treatment $\left(F_{1,18}=7.82, p<\right.$ 0.05). An abolishment of c-Fos expression induced by nicotine withdrawal in the PVN of prepro-hypocretin deficient mice was revealed by post-hoc analysis $(p<0.01)$ (Figure 2A and 2D). In the pharmacological approach, two-way ANOVA also showed a significant interaction between SB334867 and nicotine treatment $\left(F_{1,21}=4.79, p<\right.$ 0.05). Post-hoc analysis revealed a blockade of the activation of the PVN due to SB334867 pretreatment $(p<0.05)$ (Figure 2B and 2E). On the contrary, the pretreatment with TCSOX229 (10 mg/kg, ip) did not affect the activation of the PVN observed during mecamylamine-precipitated nicotine withdrawal (Figure 2C). These data indicate that the PVN is a critical region associated to both nicotine withdrawal and Hcrtr-1 activation.

CRF and AVP are the most important neuropeptides in the PVN for the regulation of the hypothalamic-pituitary-adrenal axis (25). Therefore, we investigated whether these cell types were affected during nicotine withdrawal and the regulation of this effect by the Hcrtr-1. Mecamylamine-precipitated withdrawal induced an increase of c-Fos expression in CRF-expressing neurons and SB334867 pretreatment abolished the activation in these particular cells (Figure 2G). Thus, two-way ANOVA showed a significant interaction between SB334867 pretreatment and nicotine treatment $\left(F_{1,13}=\right.$ 5.15, $p<0.05)$. Post-hoc analysis revealed the blockade of the activation of CRF neurons due to SB334867 pretreatment $(p<0.01)$ (Figure $2 \mathrm{G}$ ). In contrast, c-Fos 
expression was not significantly altered in AVP neurons during nicotine withdrawal (Figure 2H). These data indicate that the Hcrtr-1 modulates the specific activation of CRF cells in the PVN induced by the mecamylamine-precipitated nicotine withdrawal syndrome.

\section{Hypocretin neurons directly project to the hypothalamic paraventricular nucleus}

Next, we studied whether the activation of the PVN could be the result of a direct action of hypocretins on this hypothalamic nucleus. For this purpose, we performed retrograde tracing with rhodamine-labelled fluorescent latex microspheres (red beads) which were directly infused into the PVN (Figure 3B). After 7 days, retrogradely labeled neurons were observed in the lateral hypothalamic area and some of them colocalized with hypocretin-1 immunoreactivity. Of the total number of hypocretin neurons found in the representative sections obtained from the PFA/DMH $(175 \pm 14, \mathrm{n}=4), 10.6 \pm 2.6 \%$ were retrogradely labeled from the PVN, whereas $5.2 \pm 1.7 \%$ of the hypocretin cells found in the $\mathrm{LH}(201 \pm 10, \mathrm{n}=4)$ contained the retrograde tracer (Figure 3C, 3D and 3E). These results are in agreement with previous immunohistochemical studies showing the presence of hypocretin-containing fibers in the PVN $(26,27)$.

Hcrtr-1 activation in the hypothalamic paraventricular nucleus is crucial for the expression of nicotine withdrawal

We next determined if the direct infusion of the Hcrtr-1 antagonist SB334867 into the PVN could modulate the expression of nicotine withdrawal. This hypothesis was based on the Hcrtr-1 dependent activation of the PVN associated to nicotine withdrawal, the existence of direct hypocretinergic projections from the lateral hypothalamic area to the PVN, and the previously reported expression of Hcrtr-1 within the PVN $(28,29)$. The 
direct infusion of SB334867 (10 nmol/side) into the PVN before mecamylamine decreased the somatic manifestations of the nicotine withdrawal syndrome (Figure 4B). Thus, two-way ANOVA for the global withdrawal score revealed an interaction between SB334867 and nicotine treatment $\left(F_{1,31}=4.36, p<0.05\right)$. Subsequent post-hoc showed a significant reduction of the somatic signs of nicotine withdrawal in SB334867 pretreated mice $(p<0.05)$. These data indicate that the activation of Hcrtr-1 in the PVN is critically involved in the modulation of the somatic manifestations of nicotine withdrawal. Histological reconstruction of the injection sites in the PVN (Figure 4C) and a representative coronal section of the mouse brain stained with Cresyl Violet illustrating the placement of the cannula (Figure 4A) are shown. 


\section{Discussion}

Our results demonstrate that hypocretin transmission through a selective involvement of Hcrtr-1 contributes to the somatic expression of nicotine withdrawal. In addition, we reveal a crucial role of the PVN in the modulation of this effect. Thus, we show that concurrent activation of the PVN during nicotine withdrawal is dependent on hypocretin action, and that the direct blockade of the Hcrtr-1 in this nucleus decreases the severity of the physical discomfort associated to nicotine withdrawal.

In rodents, nicotine withdrawal produces somatic signs as well as affective changes similar to those observed in humans. The appearance of withdrawal symptoms following chronic nicotine cessation is an important factor precluding people from successfully quitting smoking $(30,31)$, and therapeutic measures towards the reduction of this syndrome must be addressed to maximize success rates from cessation attempts. A reduction of nicotine withdrawal was found in mice lacking the prepro-hypocretin gene. In addition, somatic nicotine withdrawal signs were attenuated in nicotine dependent mice that received the selective Hcrtr-1 antagonist SB334867, but not the Hcrtr-2 antagonist TCSOX229, before mecamylamine administration. This finding was not due to possible modifications of locomotion since the low doses of SB334867 used in this study did not induce changes in locomotor activity (20). Hypocretin receptors show different patterns of expression within the central nervous system (32) suggesting that Hcrtr-1 and -2 could differentially regulate distinct physiological functions and behaviors. Few studies to date have investigated the role of the Hcrtr-2 on addictive behaviors due to the lack of available specific Hcrtr-2 antagonists. Selective blockade of Hcrtr-2 did not affect cocaine reinstatement or intake (33), while an involvement of Hcrtr-2 in the reinforcing effects of alcohol has been recently reported (34). Our results demonstrate a specific role for Hcrtr-1 in the regulation of the somatic nicotine 
withdrawal since the Hcrtr-2 antagonist did not modify the severity of nicotine abstinence. In line with our data, previous studies have shown the ability of the Hcrtr-1 antagonist SB334867 (35) and the deletion of the prepro-hypocretin gene (36) to attenuate the somatic signs of naloxone-precipitated morphine withdrawal in mice. However, the role of Hcrtr-2 in morphine withdrawal has not been evaluated yet.

In agreement with the behavioral results, we found that nicotine withdrawal increased the recruitment of hypocretin cells within the PFA/DMH and the LH. Interestingly, blockade of Hcrtr-1 via SB334867 administration prevented the activation of hypocretin neurons in the PFA/DMH suggesting a local hypocretin signaling in the regulation of this effect. Previous studies have shown the presence of Hcrtr-1 immunoreactivity in hypocretin-containing neurons (29) and the existence of hypocretin axons making asymmetric synaptic contacts with hypocretin cells (37), which supports the idea that Hcrtr-1 may serve as a somatodendritic autoreceptor regulating the activity of hypocretin neurons.

We next investigated the possible brain areas involved in the effects of hypocretin neuronal activation given that these cells project widely through the brain. Mecamylamine-precipitated withdrawal induced an increase of c-Fos expression in the LS, the BNST and the PVN. Previous studies have described a similar induction of cFos in these brain areas following naloxone-precipitated morphine withdrawal in rats (38). LS plays an important role in the regulation of mood, motivation (39) and stress responses (40). Moreover, LS seems to participate in nicotine responses since local injection of the drug in this nucleus induces anxiety-like effects $(41,42)$, suggesting that this brain region could also be involved in the regulation of emotional states associated to nicotine withdrawal. The BNST is a component of the "extended amygdala" involved in stress and reward related behaviors (43). Nicotine, like other drugs of abuse, 
increases extracellular dopamine in the BNST (44) following acute administration. In spite of the potential role of this brain structure in nicotine abstinence, direct blockage of nicotinic acetylcholine receptors, dopamine D1-like receptors (45) or CRF1/2 receptors (46) in the BNST did not prevent the negative affective manifestations of nicotine withdrawal in rats. Notably, the activation observed in the PVN during nicotine withdrawal, but not LS and BNST activation, was prevented by the previous administration of SB334867 or prepro-hypocretin gene deletion. However, TCSOX229 pretreatment did not affect this activation, suggesting that the PVN is a crucial region connecting both nicotine withdrawal and hypocretin function through Hcrtr-1.

The PVN, which mainly controls the activity of the hypothalamic-pituitary-adrenal axis, is one of the most important integrative and regulatory centers for many behavioral, autonomic and neuroendocrine processes and several reports have emphasized its significance in the acute effects of different addictive drugs (25) including nicotine. The brain stress systems are dysregulated after repeated drug administration and withdrawal (47), which contributes to the maintenance of the addictive process. In agreement, previous evidence demonstrates that the PVN is involved in the somatic manifestations related to the stressful condition of drug withdrawal, as mainly described in the case of opiates. Thus, naloxone-precipitated morphine withdrawal induces c-Fos expression in the PVN $(38,48)$ through the increase of noradrenergic activity in this hypothalamic nucleus. The subsequent activation of the CRF system in the PVN seems to be critical for the somatic manifestations of morphine withdrawal. Recent reports indicate that CRF2 receptor activation contributes to the physical symptoms of opiate withdrawal $(49,50)$, while more inconsistent findings have been obtained in the case of the CRF1 receptor subtype (51-53). Nevertheless, the relationship between PVN activity and alterations in nicotine withdrawal-induced behaviors has not yet been assessed. Our 
results show an increase of c-Fos expression in the PVN during nicotine withdrawal, which was dependent on Hcrtr-1 activation. Consistent with this, the activation of CRFexpressing neurons associated to nicotine withdrawal within the PVN was abolished by the previous administration of SB334867. Using retrograde tracing, we demonstrated that the PVN receives direct hypocretinergic afferents from the lateral hypothalamic area, suggesting that the activation of the PVN occurs through this direct circuit. This is congruent with previous studies that showed the presence of hypocretin-containing fibers in the PVN $(26,27)$ and the well known electrophysiological effects that hypocretins exert on this hypothalamic nucleus (54). Accordingly, a depolarization of magno- and parvocellular neurons in the PVN is induced by the application of hypocretin-1 on hypothalamic slice preparations (55-57). Interestingly, we found that a high proportion of hypocretin cells projecting to the PVN come from the PFA/DMH, consistent with the previously reported moderate-strong input to the PVN from cells of the DMH (58) and the electrophysiological evidence that the PFA/DMH provides most of the local excitatory synaptic inputs to PVN neurons (59). Moreover, the Hcrtr-1 antagonist SB334867 blocked the activation of hypocretin cells in the PFA/DMH, but not in the LH, suggesting that this specific hypocretinergic pathway could play a more relevant role for the subsequent activation of the PVN during nicotine withdrawal.

Based on the regulation of the nicotine withdrawal manifestations by Hcrtr-1 and the simultaneous activation of hypocretin neurons and the PVN during this abstinence, we tested whether direct administration of SB334867 into the PVN could modulate the expression of nicotine withdrawal. Previous studies have described the presence of Hcrtr-1 in the PVN $(28,29)$. Somatic signs of nicotine abstinence were decreased after the infusion of the Hcrtr-1 antagonist into the PVN, suggesting that hypocretin signaling 
acting on Hcrtr-1 in this brain area is critically involved in the modulation of nicotine withdrawal.

In conclusion, our study reveals a novel mechanism involved in the somatic manifestations of nicotine withdrawal and advances the understanding of the role of the hypocretinergic system in the addictive properties of nicotine. Considering the crucial role of the manifestations of nicotine withdrawal in the relapse of nicotine consumption, this new function of hypocretins could have important therapeutic implications in the effort to develop new effective drugs to help smoking cessation. 


\section{Acknowledgments}

This work was supported by the Instituto de Salud Carlos III grants \#PI07/0559, \#PI10/00316, and \#RD06/001/001 (RTA-RETICS), by the Spanish Ministry of Science and Technology (Consolider-C, \#SAF2007-64062), the Catalan Government (SGR2009-00731), and by the Catalan Institution for Research and Advanced Studies (ICREA Academia program). A Plaza-Zabala is a recipient of a predoctoral fellowship from the Spanish Ministry of Education. We thank M Linares, R Martín and C Fernández for invaluable technical assistance and T Sakurai and M Yanagisawa for generously providing prepro-hypocretin knockout mice.

\section{Financial Disclosures}

The authors have no conflicts of interest to declare. 


\section{References}

1. Benowitz NL (2009): Pharmacology of nicotine: addiction, smoking-induced disease, and therapeutics. Annu Rev Pharmacol Toxicol 49:57-71.

2. Allen SS, Bade T, Hatsukami D, Center B (2008): Craving, withdrawal, and smoking urges on days immediately prior to smoking relapse. Nicotine Tob Res 10: $35-45$.

3. Hughes JR, Hatsukami D (1986): Signs and symptoms of tobacco withdrawal. Arch Gen Psychiatry 43: 289-294.

4. Hughes JR, (2007): Effects of abstinence from tobacco: valid symptoms and time course. Nicotine Tob Res 9: 315-327.

5. Malin DH, Lake JR, Newlin-Maultsby P, Roberts LK, Lanier JG, Carter VA, et al. (1992): Rodent model of nicotine abstinence syndrome. Pharmacol Biochem Behav 43: 779-784.

6. Castañé A, Valjent E, Ledent C, Parmentier M, Maldonado R, Valverde O (2002): Lack of CB1 cannabinoid receptors modifies nicotine behavioural responses, but not nicotine abstinence. Neuropharmacology 43: 857-867.

7. Jackson KJ, Martin BR, Changeux JP, Damaj MI (2008): Differential role of nicotinic acetylcholine receptor subunits in physical and affective nicotine withdrawal signs. J Pharmacol Exp Ther 325: 302-312.

8. Johnson PM, Hollander JA, Kenny PJ (2008): Decreased brain reward function during nicotine withdrawal in C57BL6 mice: evidence from intracranial selfstimulation (ICSS) studies. Pharmacol Biochem Behav 90: 409-415.

9. Berrendero F, Robledo P, Trigo JM, Martín-García E, Maldonado R (2010): Neurobiological mechanisms involved in nicotine dependence and reward: participation of the endogenous opioid system. Neurosci Biobehav Rev 35: 220-231. 
10. Salas R, Sturm R, Boulter J, De Biasi M (2009): Nicotinic receptors in the habenulo-interpeduncular system are necessary for nicotine withdrawal in mice. $J$ Neurosci 29: 3014-3018.

11. de Lecea L, Kilduff TS, Peyron C, Gao X, Foye PE, Danielson PE, et al. (1998): The hypocretins: hypothalamus-specific peptides with neuroexcitatory activity. Proc Natl Acad Sci U S A 95: 322-327.

12. Sakurai T, Amemiya A, Ishii M, Matsuzaki I, Chemelli RM, Tanaka H, et al. (1998): Orexins and orexin receptors: a family of hypothalamic neuropeptides and G protein-coupled receptors that regulate feeding behavior. Cell 92: 573-585.

13. Sakurai T, Mieda M, Tsujino N (2010): The orexin system: roles in sleep/wake regulation. Ann N Y Acad Sci 1200:149-161.

14. Bonnavion P, de Lecea L (2010): Hypocretins in the control of sleep and wakefulness. Curr Neurol Neurosci Rep 10:174-179.

15. Aston-Jones G, Smith RJ, Sartor GC, Moorman DE, Massi L, Tahsili-Fahadan P, et al. (2010): Lateral hypothalamic orexin/hypocretin neurons: A role in rewardseeking and addiction. Brain Res 1314:74-90.

16. Corrigall WA (2009): Hypocretin mechanisms in nicotine addiction: evidence and speculation. Psychopharmacology (Berl) 206: 23-37.

17. Kenny PJ (2011): Tobacco dependence, the insular cortex and the hypocretin connection. Pharmacol Biochem Behav 97: 700-707.

18. Hollander JA, Lu Q, Cameron MD, Kamenecka TM, Kenny PJ (2008): Insular hypocretin transmission regulates nicotine reward. Proc Natl Acad Sci U S A 105: 19480-19485. 
19. LeSage MG, Perry JL, Kotz CM, Shelley D, Corrigall WA (2010): Nicotine selfadministration in the rat: effects of hypocretin antagonists and changes in hypocretin mRNA. Psychopharmacology (Berl) 209: 203-212.

20. Plaza-Zabala A, Martín-García E, de Lecea L, Maldonado R, Berrendero F (2010): Hypocretins regulate the anxiogenic-like effects of nicotine and induce reinstatement of nicotine-seeking behavior. J Neurosci 30: 2300-2310.

21. Chemelli RM, Willie JT, Sinton CM, Elmquist JK, Scammell T, Lee C, et al. (1999) Narcolepsy in orexin knockout mice: molecular genetics of sleep regulation. Cell 98: 437-451.

22. Mochizuki T, Crocker A, McCormack S, Yanagisawa M, Sakurai T, Scammell TE (2004): Behavioral state instability in orexin knock-out mice. J Neurosci 24: 62916300.

23. Narita M, Nagumo Y, Hashimoto S, Narita M, Khotib J, Miyatake M, et al. (2006): Direct involvement of orexinergic systems in the activation of the mesolimbic dopamine pathway and related behaviors induced by morphine. J Neurosci 26: 398405.

24. Kovács KJ (2008): Measurement of immediate-early gene activation c-fos and beyond. J Neuroendocrinol 20: 665-672.

25. Armario A (2010): Activation of the hypothalamic-pituitary-adrenal axis by addictive drugs: different pathways, common outcome. Trends Neurosci 31: 318325.

26. Peyron C, Tighe DK, van den Pol AN, de Lecea L, Heller HC, Sutcliffe JG, et al. (1998): Neurons containing hypocretin (orexin) project to multiple neuronal systems. J Neurosci 18: 9996-10015. 
27. Date Y, Ueta Y, Yamashita H, Yamaguchi H, Matsukura S, Kangawa K, et al. (1999): Orexins, orexigenic hypothalamic peptides, interact with autonomic, neuroendocrine and neuroregulatory systems. Proc Natl Acad Sci U S A 96: 748753.

28. Hervieu GJ, Cluderay JE, Harrison DC, Roberts JC, Leslie RA (2001): Gene expression and protein distribution of the orexin-1 receptor in the rat brain and spinal cord. Neuroscience 103: 777-797.

29. Bäckberg M, Hervieu G, Wilson S, Meister B (2002): Orexin receptor-1 (OX-R1) immunoreactivity in chemically identified neurons of the hypothalamus: focus on orexin targets involved in control of food and water intake. Eur J Neurosci 15: 315328.

30. West RJ, Hajek P, Belcher M (1989): Severity of withdrawal symptoms as a predictor of outcome of an attempt to quit smoking. Psychol Med 19: 981-985.

31. Zhou X, Nonnemaker J, Sherrill B, Gilsenan AW, Coste F, West R (2009): Attempts to quit smoking and relapse: factors associated with success or failure from the ATTEMPT cohort study. Addict Behav 34: 365-373.

32. Marcus JN, Aschkenasi CJ, Lee CE, Chemelli RM, Saper CB, Yanagisawa M, et al. (2001): Differential expression of orexin receptors 1 and 2 in the rat brain. J Comp Neurol 435: 6-25.

33. Smith RJ, See RE, Aston-Jones G (2009): Orexin/hypocretin signaling at the orexin 1 receptor regulates cue-elicited cocaine-seeking. Eur J Neurosci 30: 493-503.

34. Shoblock JR, Welty N, Aluisio L, Fraser I, Motley ST, Morton K, et al. (2011): Selective blockade of the orexin-2 receptor attenuates ethanol self-administration, place preference, and reinstatement. Psychopharmacology (Berl) 215: 191-203. 
35. Sharf R, Sarhan M, DiLeone RJ (2008): Orexin mediates the expression of precipitated morphine withdrawal and concurrent activation of the nucleus accumbens shell. Biol Psychiatry 64: 175-183.

36. Georgescu D., Zachariou V., Barrot M., Mieda M., Willie JT, Eisch AJ, et al. (2003): Involvement of the lateral hypothalamic peptide orexin in morphine dependence and withdrawal. J Neurosci 23: 3106-3111.

37. Horvath TL, Diano S, van den Pol AN (1999): Synaptic interaction between hypocretin (orexin) and neuropeptide $\mathrm{Y}$ cells in the rodent and primate hypothalamus: a novel circuit implicated in metabolic and endocrine regulations. $J$ Neurosci 19: 1072-1087.

38. Núñez C, Földes A, Laorden ML, Milanes MV, Kovács KJ (2007): Activation of stress-related hypothalamic neuropeptide gene expression during morphine withdrawal. J Neurochem 101: 1060-1071.

39. Sheehan TP, Chambers RA, Russell DS (2004): Regulation of affect by the lateral septum: implications for neuropsychiatry. Brain Res Brain Res Rev 46: 71-117.

40. Singewald GM, Rjabokon A, Singewald N, Ebner K (2011): The modulatory role of the lateral septum on neuroendocrine and behavioral stress responses. Neuropsychopharmacology 36: 793-804.

41. Ouagazzal AM, Kenny PJ, File SE (1999): Stimulation of nicotinic receptors in the lateral septal nucleus increases anxiety. Eur J Neurosci 11: 3957-3962.

42. Cheeta S, Kenny PJ, File SE (2000): The role of 5-HT1A receptors in mediating the anxiogenic effects of nicotine following lateral septal administration. Eur J Neurosci 12: $3797-3802$. 
43. Jalabert M, Aston-Jones G, Herzog E, Manzoni O, Georges F (2009): Role of the bed nucleus of the stria terminalis in the control of ventral tegmental area dopamine neurons. Prog Neuropsychopharmacol Biol Psychiatry 33: 1336-1346.

44. Carboni E, Silvagni A, Rolando MT, Di Chiara G (2000): Stimulation of in vivo dopamine transmission in the bed nucleus of stria terminalis by reinforcing drugs. $J$ Neurosci 20: RC102.

45. Jonkman S, Markou A (2006): Blockade of nicotinic acetylcholine or dopamine D1like receptors in the central nucleus of the amygdala or the bed nucleus of the stria terminalis does not precipitate nicotine withdrawal in nicotine-dependent rats. Neurosci Lett 400: 140-145.

46. Marcinkiewcz CA, Prado MM, Isaac SK, Marshall A, Rylkova D, Bruijnzeel AW (2009): Corticotropin-releasing factor within the central nucleus of the amygdala and the nucleus accumbens shell mediates the negative affective state of nicotine withdrawal in rats. Neuropsycopharmacology 34: 1743-1752.

47. Koob G (2008): A role for brain stress systems in addiction. Neuron 59: 11-34.

48. Laorden ML, Núñez C, Almela P, Milanés MV (2002): Morphine withdrawalinduced c-fos expression in the hypothalamic paraventricular nucleus is dependent on the activation of catecholaminergic neurones. J Neurochem 83: 132-140.

49. Papaleo F, Ghozland S, Ingallinesi M, Roberts AJ, Koob GF, Contarino A (2008): Disruption of the CRF2 receptor pathway decreases the somatic expression of opiate withdrawal. Neuropsychopharmacology 33: 2878-2887.

50. Navarro-Zaragoza J, Núñez C, Ruiz-Medina J, Laorden ML, Valverde O, Milanés MV (2011): CRF2 mediates the increased noradrenergic activity in the hypothalamic paraventricular nucleus and the negative state of morphine withdrawal in rats. Br J Pharmacol 162: 851-862. 
51. Iredale PA, Alvaro JD, Lee Y, Terwilliger R, Chen YL, Duman RS (2000): Role of corticotropin-releasing factor receptor-1 in opiate withdrawal. J Neurochem 74: 199208.

52. Papaleo F, Kitchener P, Contarino A (2007): Disruption of the CFR/CRF1 receptor stress system exacerbates the somatic signs of opiate withdrawal. Neuron 53: 577589.

53. Navarro-Zaragoza J, Núñez C, Laorden ML, Milanés MV (2010): Effects of corticotropin-releasing factor receptor-1 antagonists on the brain stress system responses to morphine withdrawal. Mol Pharmacol 77: 864-873.

54. Winsky-Sommerer R, Boutrel B, de Lecea L (2005): The corticotrophin-releasing factor/hypocretin circuitry. Mol Neurobiol 32: 285-294.

55. Shirasaka T, Miyahara S, Kunitake T, Jin QH, Kato K, Takasaki M, et al. (2001): Orexin depolarizes rat hypothalamic paraventricular nucleus neurons. Am J Physiol Regul Integr Comp Physiol 281: R1114-R1118.

56. Samson WK, Taylor MM, Follwell M, Ferguson AV (2002): Orexin actions in hypothalamic paraventricular nucleus: physiological consequences and cellular correlates. Regul Pept 104: 97-103.

57. Follwell MJ, Ferguson AV (2002): Cellular mechanisms of orexin actions on paraventricular nucleus neurones in rat hypothalamus. J Physiol 545: 855-867.

58. Qi Y, Namavar MR, Iqbal J, Oldfield BJ, Clarke IJ (2009): Characterization of the projections to the hypothalamic paraventricular and periventricular nuclei in the female sheep brain, using retrograde tracing and immunohistochemistry. Neuroendocrinology 90: 31-53.

59. Boudaba C, Schrader LA, Tasker JG (1997): Physiological evidence for local excitatory synaptic circuits in the rat hypothalamus. J Neurophysiol 77: 3396-3400. 
Plaza-Zabala et al.

60. Paxinos G, Franklin KBJ (1997): The mouse brain in stereotaxic coordinates. San Diego Academic Press. 


\section{Figure legends}

Figure 1. Hypocretin transmission through the specific activation of Hcrtr-1 modulates the expression of the nicotine withdrawal syndrome. Acute mecamylamine $(2 \mathrm{mg} / \mathrm{kg}$, sc) was administered to precipitate nicotine withdrawal in $\mathrm{C} 57 \mathrm{BL} / 6 \mathrm{~J}$ mice receiving a continuous perfusion of nicotine $(25 \mathrm{mg} / \mathrm{kg} / \mathrm{day})$ from subcutaneously implanted osmotic minipumps. SB334867 and TCSOX229 were administered 30 min before mecamylamine. (A,B,C) A global withdrawal score was calculated for each animal by giving each individual sign a relative weight in (A) wildtype or prepro-hypocretin knockout ( $n=9-14$ mice per group) mice, in (B) vehicle or SB334867 (5 and $10 \mathrm{mg} / \mathrm{kg}$, ip) pretreated C57BL/6J mice ( $n=9-24$ mice per group) and in (C) vehicle or TCSOX229 (5 and $10 \mathrm{mg} / \mathrm{kg}$, ip) pretreated C57BL/6J mice $(n=$ 8-12 mice per group). (D) Schematic anatomic representation of lateral hypothalamic area subdivisions adapted from Paxinos and Franklin's stereotaxic atlas (60). Colored areas delineate regions where c-Fos expression was examined. (E,F) Percentage of cFos-positive hypocretin-1-expressing neurons in vehicle, SB334867 (5 mg/kg, ip) or TCSOX229 (10 mg/kg, ip) pretreated C57BL/6J mice ( $n=6-13$ mice per group) in the perifornical and dorsomedial hypothalamus (PFA/DMH) and in the lateral hypothalamus (LH). (G) Representative images of sections of the PFA/DMH obtained by confocal microscopy after direct double labeling combining rabbit polyclonal antiserum to c-Fos (red) with mouse monoclonal antibody to hypocretin-1 (green). Arrowheads indicate c-Fos-positive hypocretin-1-expressing neurons. Scale bar, 100 $\mu \mathrm{m}$. Data are expressed as mean \pm S.E.M. $\star p<0.05, \star \star p<0.01$ compared to the control group; $\# p<0.05, \# \# p<0.01$ comparison between pretreatments or genotypes. 


\section{Figure 2. Hypocretins acting on hypocretin receptor-1 regulate the activation of} the hypothalamic paraventricular nucleus associated to nicotine withdrawal. $(\mathbf{A}, \mathbf{B}, \mathbf{C})$ c-Fos expression in the PVN $2 \mathrm{~h}$ after precipitation of nicotine withdrawal by acute mecamylamine (2 mg/kg, sc) injection in (A) wild-type or prepro-hypocretin knockout mice ( $n=5-6$ mice per group), in (B) vehicle or SB334867 (5 mg/kg, ip) pretreated C57BL/6J mice ( $n=5-8$ mice per group) and in (C) vehicle or TCSOX229 (10 $\mathrm{mg} / \mathrm{kg}$, ip) pretreated C57BL/6J mice ( $n=7-9$ mice per group). SB334867 and TCSOX229 were administered $30 \mathrm{~min}$ before mecamylamine. (D,E) Representative images of the PVN obtained by confocal microscopy after direct labeling with rabbit polyclonal antiserum to c-Fos in SB334867 pretreated and knockout mice. Scale bar, $100 \mu \mathrm{m}$. (F) Schematic anatomic representation of the PVN adapted from Paxinos and Franklin's stereotaxic atlas (60). The colored area delineates the region where c-Fos expression was examined. (G,H) Percentage of CRF (G) and AVP (H) cells expressing c-Fos in the PVN $2 \mathrm{~h}$ after precipitation of nicotine withdrawal by mecamylamine (2 $\mathrm{mg} / \mathrm{kg}, \mathrm{sc})$ in vehicle or SB334867 (5 mg/kg, ip) pretreated C57BL/6J mice $(n=3-6$ mice per group). Representative images of c-Fos expressing CRF neurons in the PVN are shown in Figure S4 in Supplement 1. Data are expressed as mean \pm S.E.M. $\star p<$ $0.05, \star \star p<0.01$ compared to the control group; \#p $<0.05, \# \# p<0.01$ comparison between pretreatments or genotypes.

Figure 3. Hypocretin neurons directly project to the hypothalamic paraventricular nucleus. (A) Schematic diagrams adapted from the mouse brain atlas of Paxinos and Franklin (1997) showing the representative level of the injection site of the red fluorescent latex microspheres (Bregma $-0.82 \mathrm{~mm}$ ) and the representative level where retrogradely labeled neurons were analyzed (Bregma $-1.70 \mathrm{~mm}$ ). (B) Representative 
merged brightfield and immunofluorescence image of the injection site. Red latex microsphere deposits are visible in the PVN. Scale bar, $100 \mu \mathrm{m}$. (C,D) Representative confocal images of mouse brain sections containing retrogadely labeled neurons (red) of the perifornical and dorsomedial hypothalamus (PFA/DMH) after direct labeling with mouse monoclonal antibody to hypocretin-1 (green). Arrowheads indicate retrogradely labeled hypocretin expressing neurons. Scale bar, $100 \mu \mathrm{m}$. (D) Higher magnification of the dual labeled neurons in the PFA/DMH. Scale bar, $50 \mu \mathrm{m}$. (E) The percentage of hypocretin-1 expressing neurons that were retrogradely labeled with the tracer were quantified in lateral hypothalamic sections. Data are expressed as mean \pm S.E.M. $\star p<$ 0.05 comparison between the percentage of hypocretin neurons that project from the PFA/DMH and LH to the PVN.

Figure 4. Hypocretin receptor-1 activation in the hypothalamic paraventricular nucleus is crucial for the expression of nicotine withdrawal. (A) A representative photomicrograph of a coronal section of a mouse brain stained with cresyl violet showing a cannula placement in the PVN. (B) Mecamylamine-precipitated nicotine withdrawal in C57BL/6J mice after direct infusion of SB334867 (10 nmol/side) into the PVN. Withdrawal was precipitated by acute mecamylamine administration $(2 \mathrm{mg} / \mathrm{kg}$, sc) after 14 days of nicotine perfusion ( $25 \mathrm{mg} / \mathrm{kg} / \mathrm{day}$ ) by subcutaneous minipumps. SB334867 was administered 30 min before mecamylamine. A global withdrawal score was calculated for each animal by giving each individual sign a relative weight in vehicle or SB334867 pretreated C57BL/6J mice ( $n=7-13$ mice per group). (C) Schematic diagrams adapted from the mouse brain atlas of Paxinos and Franklin (1997) showing representative injection sites in the PVN. Black dots indicate locations of cannula tips. Each image indicates the distance from Bregma. Data are expressed as 
Plaza-Zabala et al.

mean \pm S.E.M. $\star \star p<0.01$ compared to the control group; $\# p<0.05$ comparison between pretreatments. 